\title{
INSIGHT: Internet-Sensor Integration for Habitat Monitoring
}

\author{
Murat Demirbas Ken Yian Chow Chieh Shyan Wan \\ Dept. of Computer Science \& Engineering \\ University at Buffalo \\ Buffalo, NY 14226 \\ \{demirbas, kenchow, chiehwan\}@cse.buffalo.edu
}

\begin{abstract}
We present our experience with designing, developing, and deploying of an Internet accessible wireless sensor network for monitoring of the temperature, humidity, and illumination of a controlled environment. The design goals for our system, INSIGHT: INternet-Sensor InteGration for HabitaT monitoring, are extended deployment lifetime, remote querying and configuration, ease of deployment, and reliability. We present results from our deployment of INSIGHT in an apartment and a greenhouse.
\end{abstract}

\section{Introduction}

Wireless sensor networks (WSN) are useful in many monitoring applications [7, 9, 10]. As it is becoming increasingly more feasible to deploy wireless sensor nodes for ubiquitous and high-fidelity monitoring, WSN has the potential to improve Supervisory Control and Data Acquisition (SCADA) [18] systems used for monitoring and control of a plant in industries such as telecommunications, water and waste control, energy, and transportation.

As a step, towards the development of an industrial strength monitoring system, we investigate monitoring of a controlled, small environment via WSN. We see the following as the core set of requirements.

1. Energy efficiency: The sensor nodes should not need refreshing of batteries for at least 6 months.

2. Remote querying and reconfigurability: It should be possible to query the data in several formats and to reconfigure the monitoring parameters via the Internet.

3. Ease of deployment: The client should be able deploy the system without any need for special configuration.
4. Reliability: The provided data should be real-time and high-fidelity. The system should be available $99 \%$ of the time and should recover from a crash quickly.

Our Contributions. Here we discuss our experience with designing, developing, and deploying of a system, INSIGHT: INternet-Sensor InteGration for HabitaT monitoring, that fulfils the above requirements.

To enable energy efficiency and ease of deployment we use a single-hop network for INSIGHT. Without the need for forwarding messages from other nodes, the nodes do not need to stay awake or coordinate to wake up. In our case, each node occasionally wakes up only to send a report to the master, the base station, and spends the rest of the time in sleep mode to save energy. We also implemented a "delta reporting" technique [16] that enables both fast reaction time to changes in sensor readings as well as energy-efficiency when sensed values do not differ much from previous readings. INSIGHT can achieve about 6 months lifetime (using standard AA batteries) by sampling sensors every minute. Also due to our single-hop network decision, the deployment of the network is as easy as turning on a node and dropping it some place for monitoring.

To enable remote querying, we maintain a webserver and an SQL database at the base station. Users simply type in the base station's web address into their internet browser to query for data. Data is available for extraction through an XML front-end on the webserver or in the form of a TinyML query [2]. Using the website, sensor data from motes can be compared with each other, and users can also subscribe to get email alerts when a sensor reading has exceeded a threshold configured via the web interface. Moreover, the client can reconfigure the monitoring parameters, such as the sampling frequency and the delta thresholds, through the web interface. INSIGHT is currently deployed in a greenhouse and the website is accessible at http://INSIGHT.podzone.net for querying. 
In order to maintain a high level of reliability, we keep the system as simple as possible. We use a watchdog timer for the nodes and the laptop to recover from crashes and remote login capability to change webserver settings.

We study two deployments in this paper. The first is in an apartment while the second deployment is in the Dorsheimer Greenhouse at the University at Buffalo. Through these deployments, we demonstrate INSIGHT's energy efficiency, reliability, ease of use, and remote querying capabilities. Our deployment at the greenhouse surfaced some concerns with overheating that may be harmful to the experiments the scientist has been running on the tobacco plants.

Related work. Due to the industrial significance of greenhouse monitoring, Crossbow offers a WSN solution to this end that aggregates sensed data in a database and uses MATLAB as the front-end for querying [17]. In contrast to the Crossbow's solution, INSIGHT is fully Internet integrated and enables querying and graph generation via a web interface, as well as allowing reconfiguration of the monitoring parameters, and subscriptions to alerts based on specified thresholds.

The idea of using a tiered architecture is not new. TENET [19] suggests an architecture where sensor nodes are responsible only for data gathering, and more powerful tier-2 nodes, such as Stargates [17], are responsible for storing and managing the data as well as retasking the WSN. In contrast to TENET which allows multihop WSN, we emphasize the benefits of using a single-hop WSN architecture as it simplifies the WSN software significantly as well as prolonging the lifetime of nodes.

TinyDB [16] introduced the delta reporting technique we use for energy conservation. TinyDB uses a multihop WSN and coordinates the sleep patterns of the nodes for data exfiltration from the WSN. However, TinyDB does not run on mica 2 or TelosB mote architectures. In contrast to TinyDB, INSIGHT provides Internet integration at the basestation.

Outline of the rest of the paper. After presenting the system architecture in Section 2 we discuss our methods for energy efficiency in Section 3, methods for reliability in Section 4, and Internet integration in Section 5. In Sections 6 and 7, we present results from our deployments at an apartment and a greenhouse, and we conclude the paper in Section 8.

\section{System Architecture}

INSIGHT follows a simple system layout. All sensor nodes send their sensor readings to the base station, and the base station serves those data to users via the Internet.

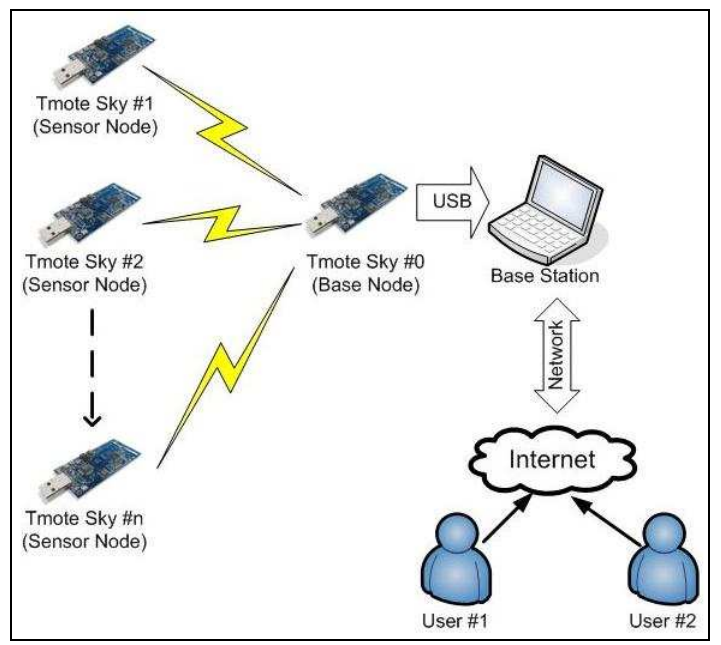

Figure 1. INSIGHT system overview

The base station is responsible for aggregating all the sensors data, converting the raw data into meaningful values and storing them into a database. Users can access those data by visiting a webpage on the server or running an XML query and then extracting the result from the base station.

Our sensor network provides four sensor readings: humidity, temperature, photosynthetically active radiation (PAR) light, and internal voltage. The data sample from the PAR sensors is the light reading that is useful for plants and the internal voltage is used for monitoring the battery power on the mote itself.

\subsection{Sensor network}

Our sensor network uses the Tmote-Sky hardware manufactured by MoteIV [6]. The sensor nodes (motes) come in matchbox-size printed circuit boards. Each mote has an $8 \mathrm{MHz}$ Texas Instruments MSP430 microcontroller (10k RAM, 48k Flash) and a 250kbps 2.4GHz IEEE 802.15.4 Chipcon CC2420 Wireless Transceiver which is Zigbee ready [13]. The fielddeployed motes also have integrated humidity, temperature, and light sensors on board. Humidity and temperature is measured using Sensirion's SHT11 [14] sensor while light is measured using Hamamatsu's S1087 PAR light sensor [15]. Motes use two standard AA batteries for power.

To keep the architecture simple, INSIGHT uses single-hop communications between the motes and the base station. The $\mathrm{CC} 2420$ radios have a communication range of approximately 100 meters, which is well beyond the size of most of our target deployment environment. We use the TinyOS version 1.1.14 [1] distribution as a development framework for the motes firmware. To have 
the motes sample and send the data via wireless communication, we modified the Oscilloscope program provided by Moteiv to optimize the sensor reading and sampling rate to fit our application. TinyOS's communications module for Tmote uses the Berkeley Media Access Control (B-MAC) [4].

\subsection{Base station}

The base station provides the interface between users and the WSN by collecting sensor readings, managing the database, and enabling remote querying and reconfiguration as well as supporting subscriptions to alerts. We chose to use a portable computer as it is simpler to develop and run the base station using a portable computer as opposed to a palm device or application-specific gateway [17]. One of the motes connects to the USB port of the laptop and act as a gateway. The laptop came equipped with an 802.11g wireless card and is able to connect to any nearby wireless access point that provides access to the Internet. The laptop is setup to use Dynamic Host Configuration Protocol to obtain an IP address automatically either from the wireless access point or through the Ethernet. Figure 2 lists the software running on the basestation.

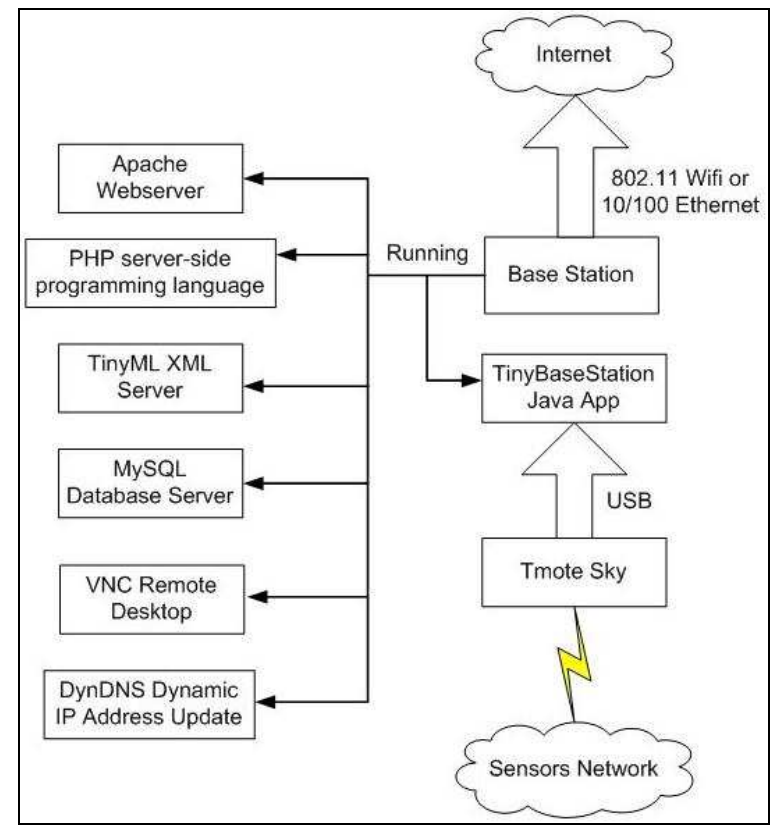

Figure 2. Basestation system overview

\section{Energy Efficiency}

One of the goals of INSIGHT is to prolong the lifetime of the sensor nodes by putting them to sleep when they are idle. Recent versions of TinyOS (version
1.1.14 and onwards) have support for a Hardware Presentation Layer (HPL) for the CC2420 radio. The HPL power management module puts the mote to sleep when certain conditions are met: 1) the radio is turned off, 2) SPI (Serial Peripheral Interface) interrupt is disabled, 3) task queue is empty, and 4) high speed clock's output-compare-interrupts are disabled [1]. Our system uses the GenericComm module, which provides the HPL power management control interface. When a mote is idle, we explicitly turn off its radio for the HPL to put the mote to sleep. The result with the power management is significant as seen in Table 1 . When the radio is off the current consumption is 0.27 milliamps with HPL versus $22 \mathrm{~mA}$ without HPL, indicating a saving by a factor of 100. Also when the radio is on consumption with HPL is $20 \mathrm{~mA}$ compared to $29 \mathrm{~mA}$ without HPL. Figure 3 shows a current measurement taken on a mote running without the HPL power saving code with the radio turned off. Thanks to our single-hop network, when a mote does not have any data to report it can sleep without the need to wake up to forward messages from other nodes.

Table 1. Comparison of Energy Consumption with different firmware

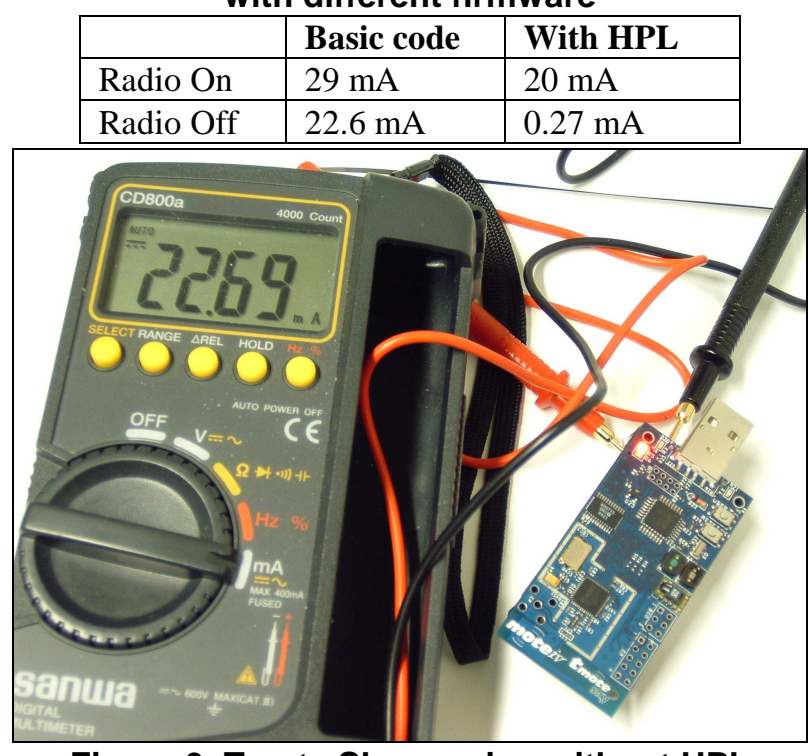

Figure 3. Tmote Sky running without HPL

In addition to the HPL power saving module, INSIGHT has also implemented the delta reporting mechanism (similar to TinyDB's technique [16]) to prolong the sensor's active lifetime. In each duty cycle, the sensor calculates the changes between the current sample and the cumulative average; if the change is within the acceptable delta range, the mote goes back to sleep without reporting to the base station. However, if the change exceeds the expected delta range, the mote turns on the radio and sends the sampled reading to the 
base station. Each duty cycle is set to 1 minute, and after 20 rounds of duty the cumulative average readings are reported to the base station as a heartbeat message, and the average is reset. The improvements due to the delta reporting are presented in Sections 6 and 7.

By comparing the sensed reading to the average, INSIGHT is highly sensitive to a change in sampled readings. By default, INSIGHT is set to report sample readings if the raw humidity reading differs by more than $8 \%$ (roughly $1 \%$ to $2 \%$ calibrated) from the average, raw temperature reading differs by $2 \%$ (roughly 0.2 Celcius), raw PAR data changed by more than $5 \%$ (roughly 2 Lux) or a $1 \%$ change (roughly 0.03 volts) in internal voltage reading.

\section{Reliability}

Here we discuss our experience with the reliability of INSIGHT in our deployments.

During one of our experimental deployments (before the introduction of "delta reporting") we have noticed that one of the sensor nodes stopped sending messages to the base station. More specifically, we found that even though the node wakes up and samples data, it fails to transmit any message. Also, we had noticed a peculiar failing mode occasionally at the other nodes. We had programmed the motes to toggle the LED before sampling and toggle it again after "Sampling_Done" event is raised. Since initially LED is off this would lead to nodes turning on LED shortly only during sampling phase. However, we saw that some of the nodes had LED on most of the time, which is turned off only shortly during what appears to be the sampling phase. On further investigation we have concluded that the LED problem was due to loss of a "Sampling_Done" event after which the LED started to work out of phase with the sampling.

From the LED observation, we decided that the node whose radio is stuck in the earlier experiment might have had the same problem. Due to loss of a "Send_Done" event the "pending" bit of the radio would always remain TRUE, preventing the node from transmitting any further messages. We addressed this issue by using a timer to reset the "pending" bit, in case it fails to be cleared by the "Send_Done" event. We have not witnessed the node failure after this fix.

We also implemented a watchdog timer to recover from cases where a mote 'freezes' and fails to perform any further tasks due to a deadlock in the tasks queue. This timer has to be reset every time it wakes up and queries the onboard sensors. If for any reason this timer is left running for too long, its overflow interrupt will force a soft reset on the motes. Our default grace period for a reset is 4 duty cycles (i.e. 4 minutes).
In our deployments, we have not witnessed the webserver, database server or the operating system crash. However, we have provided a watchdog timer script that resets the webserver and the database server in case they become unresponsive.

\section{Internet Integration}

One of our significant contributions with INSIGHT is the integration of a WSN with the Internet. Our focus here is on the ease of use and ease of deployment.

Ease of deployment: INSIGHT is designed to operate and provide environmental data to the outside world with only minimal user intervention. The base station is setup such that all essential applications launch themselves automatically on startup. The Serial Forwarder and Base Station software runs upon boot up, the Apache webserver begins serving webpages immediately and users can locate the webpage of the base station by navigating to a dynamic DNS address [8] which detects and updates that address to point to the current IP address obtained by the base station's network interface. MySQL server is installed on the base station to provide a database server for storing motes information (e.g. description, location, etc.) and sensors data. Sensor data is timestamped as it arrives in the database. The system can basically be up and running by just turning on all the motes and the base station.

Ease of use: There are two ways for a user (a human or a program) to access and query the WSN. The first is that the user can access and retrieve data by visiting the website on the base station. Alternatively, we also provide an XML interface for data extraction. For the XML part, data can be queried using TinyML [2] or through the web interface. The results of the query are placed on an XML file available via the webserver.

In line with keeping the user interface simple but powerful, the website ${ }^{+}$is divided into three parts:

1. Graphical Overview - Provides access to the data by using graphs ${ }^{++}$.

2. Tactical Overview - Provides pseudo real-time access to the data with mote locations marked on a top-view image.

3. Query Wizard - The wizard asks a question and the user select the options desired. The data is then returned based on the user's choice.

By accessing any of the 3 sections on the website, we believe INSIGHT can cater to a wide range of requests from a simple overview to detailed querying of each sensor. The website is at http://INSIGHT.podzone.net/.

+ The website design is due Luka Cvrk [ http://www.solucija.com ] ++ Graphs generated using JPGraph [http://www.aditus.nu/jpgraph] 


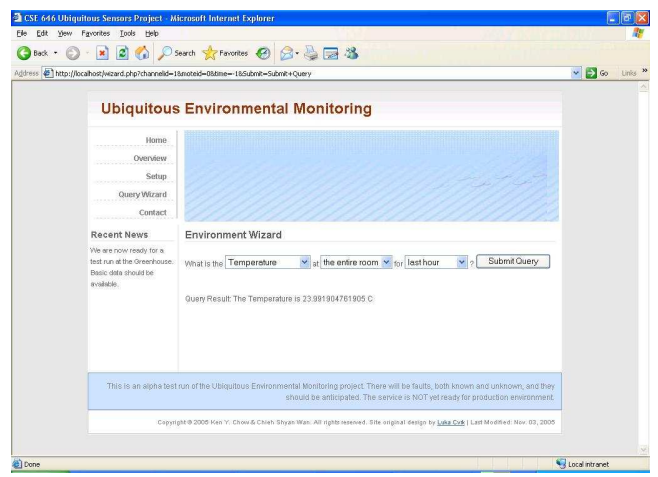

Figure 4. Query Wizard screenshot

\section{Apartment deployment results}

Before the greenhouse deployment, several trial deployments were performed in an apartment. During these deployments, we studied the usability, reliability and energy consumption aspects of INSIGHT. We had several interesting observations. For example, in certain areas of the apartment, it was cold while other areas were warmer even though these areas are less than 10 feet away. This indicated a weak spot within the apartment and that heating is not efficient enough. Figure 5 shows this observation, taken on Nov. $11^{\text {th }}, 2005$ in the apartment.

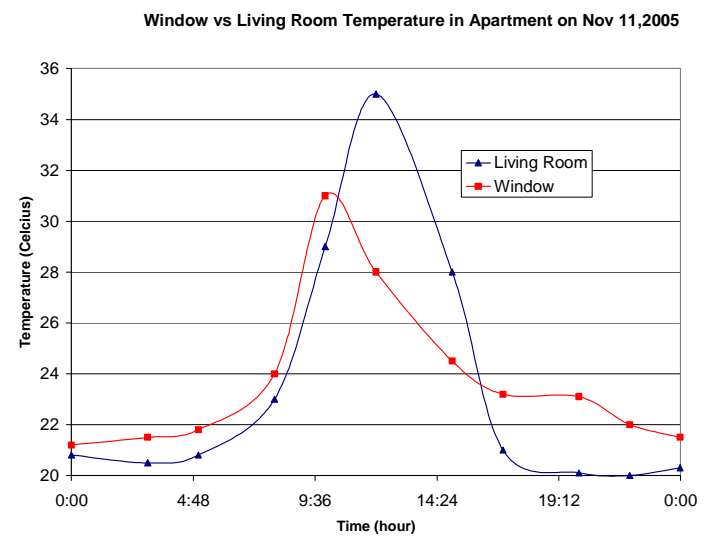

Figure 5. Window v.s. living room temperature in Apartment on Nov. 11, 2005

The energy conservation observed from using Delta monitoring was significant. Theoretically, a mote can still operate at $1.8 \mathrm{~V}$ from $2 \mathrm{AA}$-sized batteries [6]. Running at $0.25 \mathrm{~mA}$ constant current consumption will give about 4300 service hours on 2 standard AA batteries. This translates into roughly 180 days of operation for the motes. In the worst case (i.e. a volatile environment), the motes will transmit data every minute. For approximately 0.5 second per minute of the time, the motes turn their radio on, while the remaining 59.5 seconds per minute is off. With this consumption rate, the motes will still be able to operate for about 160 days. Due to recent improvements in the manufacturing of batteries, Lithium cells became available in AA size off-the-shelf. A single Lithium AA-sized battery can operate the mote for up to 9000 service hours (more than a year) with $0.25 \mathrm{~mA}$ constant current consumption [12].

\section{Greenhouse deployment result}

We have deployed INSIGHT in the Dorsheimer Greenhouse at the University at Buffalo to help a scientist closely monitor the environmental conditions in the greenhouse while she is running experiments on growth rate of tobacco plants. Figure 7 shows the locations for mote deployment, which were picked by the laboratory personnel. Due to the relatively small size of the bay, there were no disconnected or unreliable links between the motes and the base station.

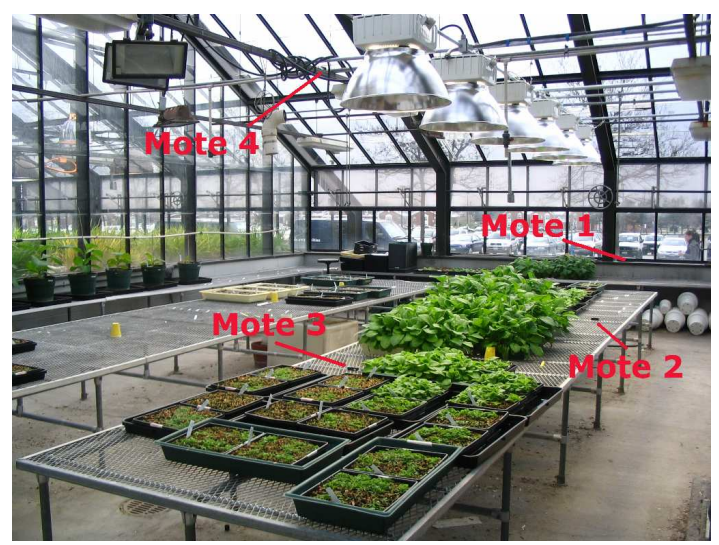

Figure 7. Deployment location of the motes

After a twenty-day deployment, the battery lives on the motes were still very good. We observed an average of about 0.15 volts drop in the battery. Since motes are able to survive until the operating voltage falls below $1.8 \mathrm{~V}$, we can afford a drop of $1.2 \mathrm{~V}$ from a fresh set of batteries. $1.2 \mathrm{~V}$ divide by $0.15 \mathrm{~V}$ multiply with 20 days will yield 160 days. This is in line with our expectation that a mote can survive for 160-180 days before running out of batteries. A comparison of the motes with and without Delta Monitoring is shown in Figure 9. We observe that Delta Monitoring improves the lifetime significantly.

By placing the motes strategically, the scientist managed to identify locations that are wasting heat. Comparing a mote placed in the center and a mote placed about 5 feet higher (Figure 8), there was already a 2 degrees Celsius difference. Besides the large difference within the greenhouse, overall temperature was also fluctuating significantly. The ideal temperature should be 
around $27^{\circ} \mathrm{C}$, but we observed variations from as low as $15^{\circ} \mathrm{C}$ to as high as $49{ }^{\circ} \mathrm{C}$ (Table 2). According to the scientist, the plants within the greenhouse may be at risk if left above $40{ }^{\circ} \mathrm{C}$ for over 1 hour. The reason for the overheating is due to the air conditioning being switched off by facility management during winter months.

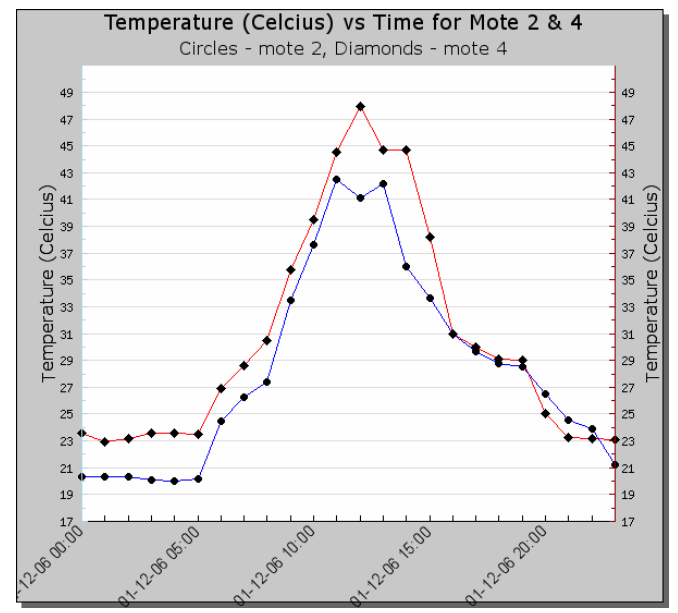

Figure 8. Mote 2 (center) vs Mote 4 (elevated) temperature comparison on Jan 12

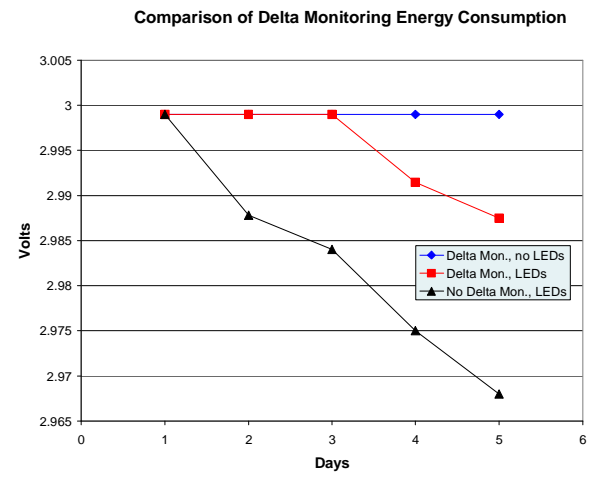

Figure 9. Voltage comparison for firmwares

Also, of interest is the observation that the temperature and humidity in the greenhouse are often inverse of each other (refer to Figure 10 \& 11). Given a temperature, the humidity is highly predictable and vice versa. At certain times though, temperature and humidity appear to both drop. This is due to the heater being turned on, which reduces humidity dramatically.

The scientist using INSIGHT to monitor the greenhouse found that our system was easy to deploy and use. She was especially happy with INSIGHT's thorough and high-fidelity monitoring data which she can study and use to make her case to facility management about several problems she has been facing with the operation of the greenhouse.
Table 2. Average, Low \& High Temperature in the Greenhouse from Jan 3 through Jan 15

\begin{tabular}{|l|c|c|c|}
\hline & Average $\left({ }^{\mathbf{}} \mathbf{C}\right)$ & Low $\left({ }^{\circ} \mathbf{C}\right)$ & High $\left({ }^{\circ} \mathbf{C}\right)$ \\
\hline $1 / 3 / 2006$ & 23.03 & 15.01 & 30.30 \\
\hline $1 / 4 / 2006$ & 21.91 & 14.74 & 25.94 \\
\hline $1 / 5 / 2006$ & 23.28 & 19.03 & 27.32 \\
\hline $1 / 6 / 2006$ & 27.53 & 17.96 & 40.60 \\
\hline $1 / 7 / 2006$ & 23.87 & 17.62 & 31.98 \\
\hline $1 / 8 / 2006$ & 30.20 & 18.22 & 42.32 \\
\hline $1 / 9 / 2006$ & 24.51 & 18.77 & 29.28 \\
\hline $1 / 10 / 2006$ & 26.39 & 18.46 & 37.29 \\
\hline $1 / 11 / 2006$ & 30.09 & 18.20 & 46.67 \\
\hline $1 / 12 / 2006$ & 34.86 & 19.88 & 49.56 \\
\hline $1 / 13 / 2006$ & 28.02 & 20.47 & 37.86 \\
\hline $1 / 14 / 2006$ & 23.53 & 15.48 & 31.96 \\
\hline $1 / 15 / 2006$ & 29.62 & 10.61 & 48.34 \\
\hline
\end{tabular}

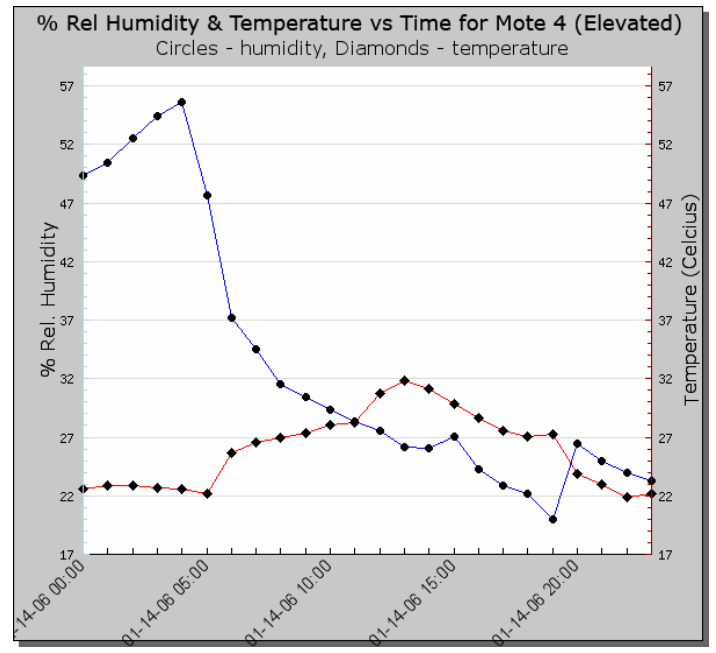

Figure 10. Humidity \& Temperature comparison on Jan 14 for Mote 4 (Elevated)

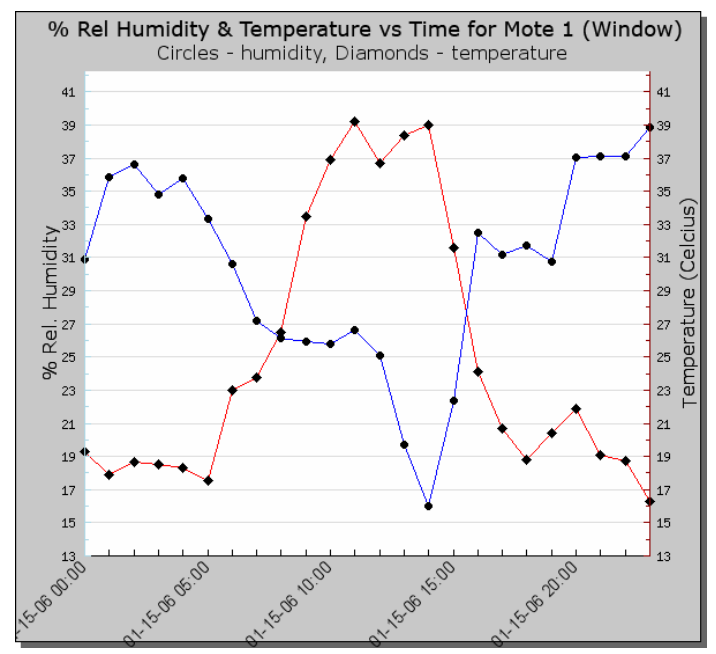

Figure 11. Humidity \& Temperature comparison on Jan 15 for Mote 1 (Window) 


\section{Concluding Remarks}

In this paper we reported on our experience with designing, developing, and deploying of INSIGHT. INSIGHT enables remote querying and reconfiguration of a WSN via Internet and subscribing to alerts based on user specified threshold over the web. INSIGHT is energy-efficient; the batteries on the sensor nodes do not need replacing for about 6 months (and with Lithium batteries, over a year). Furthermore, the sensor network's fidelity (i.e. sensitivity to environmental changes) can be set so that it is highly responsive yet also energy efficient. Finally, INSIGHT does not require pre-configuration, turning on the sensor nodes and the base station is enough for deployment. For office environments where a PC is available, the PC can be used as a base station, reducing the cost of INSIGHT to just the cost of sensor nodes, which is about $\$ 100$ each. With all these to its advantage, we hope that INSIGHT can serve as a stepping-stone for industrial quality WSN monitoring systems that can help improve on the existing SCADA systems.

We believe that a single-hop network architecture is a better choice than providing multi-hop capability in the WSN. Using single-hop network architecture improves energy-efficiency as nodes do not need to stay awake to receive broadcasts from other nodes for forwarding, facilitates the development as implementation of complex services (such as routing, time-synchronization, and coordination) can be avoided, and enables reliability through simplicity. Therefore, for monitoring a large area where one single-hop WSN is inadequate, we believe that, from the holistic system design perspective, using multiple base stations with single-hop WSN associated with each is a better choice than using a single base station with a multi-hop WSN.

In our experience, enabling Internet accessibility enhanced the usability of the WSN greatly. As most users are familiar with the Internet, they find our web-based user interface easy to understand. Also due to the ubiquity of Internet, users can conveniently access INSIGHT functionality such as querying, reconfiguration, and subscribing to alerts from anywhere, anytime.

In future work, we will improve INSIGHT's functionality by integrating intrusion detection and actuator/control mechanisms using off-the-shelf hardware components. To improve energy-efficiency further, we will investigate a predictive monitoring technique similar to that in [3]. Finally, we will consider hardware based watchdog timer solutions to address an OS crash at the base station. Since all sensor data is saved upon arrival at the base station, and since the INSIGHT software starts automatically on boot-up a single-reset of the base station could be sufficient for our purposes.
Acknowledgment: We would like to thank Dr. Margaret Hollingsworth for collaborating with us to use her greenhouse bay at the Dorsheimer Greenhouse Laboratory as a test site, and for insightful comments that helped us improve our system.

\section{References}

[1] TinyOS Tutorial: www.tinyos.net

[2] N. Ota, WTC Kramer, "TinyML: Meta-data for Wireless Networks", Technical Report, University of California, Berkeley, Computer Science Dept, 2003.

[3] S. Goel and T. Imielinski, "Prediction-based monitoring in sensor networks: Taking lessons from MPEG", Computer Comm. Review, 31(5), Oct. 2001.

[4] J. Polatre, J. Hill, and D. Culler, "Versatile low power media access for wireless sensor networks", In ACM SenSys, 2004.

[5] Ilyas, editor, Handbook of Sensor Networks:. Compact Wireless and wired sensing systems, CRC Press, 2003.

[6] Moteiv Tmote Brochure/Datasheet: www.moteiv.com

[7] B. Tolle, J. Polastre, R. Szewczyk, D. Culler, N. Turner, K. Tu, S. Burgess, T. Dawson, P. Buonadonna, D. Gay and W. Hong, "A Macroscope In The Redwoods", SenSys, California, 2005

[8] PL Tsai, CL Lei and WY Wang, "A remote control scheme for ubiquitous personal computing", 2004 IEEE International Conference on Networking, Sensing and Control, 2004.

[9] A. Mainwaring, J. Polastre, R. Szewczyk, D. Culler, and J. Anderson, "Wireless Sensor Networks for Habitat Monitoring", WSNA'02, Atlanta, Georgia, September 2002

[10] J. Burrell and T. Brooke and R. Beckwith, "Vineyard Computing: Sensor Networks in Agricultural Production", IEEE Pervasive Computing, 2004.

[11] Energizer e2 datasheet: data.energizer.com

[12] Tadiran Lithium Ion datasheet: www.tadiranbat.com

[13] Chipcon CC2420 datasheet: www.chipcon.com

[14] Sensirion SHT11 datasheet: www.sensirion.com

[15] Hamamatsu S1087 datasheet: www.hamamatsu.com

[16] S. Madden, J. Hellerstein and W. Hong, "TinyDB: In-Network Query Processing in TinyOS", telegraph.cs.berkeley.edu/tinydb , 2003.

[17] Stargate datasheet: www.xbow.com

[18] L. Tan and K. Taylor, "Mobile SCADA with Thin Clients A Web Demonstration", ICITA, Bathurst, Australia, 2002.

[19] R. Govindan, E. Kohler, D. Estrin, F. Bian, K. Chintalapudi, O. Gnawali, S. Rangwala, R. Gummadi and T. Stathopoulos, "Tenet: An Architecture for Tiered Embedded Networks", CENS Technical Report 56, 2005 